\title{
Immediate Reward Bias in Humans: Fronto-Parietal Networks and a Role for the Catechol-O-Methyltransferase $158^{\text {Val/Val }}$ Genotype
}

\author{
Charlotte A. Boettiger, ${ }^{1,2,3}$ Jennifer M. Mitchell, ${ }^{1}$ Venessa C. Tavares, ${ }^{1}$ Margaret Robertson, ${ }^{1}$ Geoff Joslyn, ${ }^{1}$ \\ Mark D’Esposito, ${ }^{4}$ and Howard L. Fields ${ }^{1,2}$ \\ ${ }^{1}$ Ernest Gallo Clinic and Research Center, Emeryville, California 94608, ${ }^{2}$ Department of Neurology, University of California, San Francisco, San Francisco, \\ California 94143, ${ }^{3}$ Department of Psychology, Biomedical Research Imaging Center, and Curriculum in Neurobiology, University of North Carolina, Chapel \\ Hill, North Carolina 27599-3270, and ${ }^{4}$ Helen Wills Neuroscience Institute and Department of Psychology, University of California, Berkeley, California
} 94720

The tendency to choose lesser immediate benefits over greater long-term benefits characterizes alcoholism and other addictive disorders. However, despite its medical and socioeconomic importance, little is known about its neurobiological mechanisms. Brain regions that are activated when deciding between immediate or delayed rewards have been identified (McClure et al., 2004, 2007), as have areas in which responses to reward stimuli predict a paper-and-pencil measure of temporal discounting (Hariri et al., 2006). These studies assume "hot" and "cool" response selection systems, with the hot system proposed to generate impulsive choices in the presence of a proximate reward. However, to date, brain regions in which the magnitude of activity during decision making reliably predicts intertemporal choice behavior have not been identified. Here we address this question in sober alcoholics and non-substance-abusing control subjects and show that immediate reward bias directly scales with the magnitude of functional magnetic resonance imaging bold oxygen leveldependent (BOLD) signal during decision making at sites within the posterior parietal cortex (PPC), dorsal prefrontal cortex (dPFC), and rostral parahippocampal gyrus regions. Conversely, the tendency of an individual to wait for a larger, delayed reward correlates directly with BOLD signal in the lateral orbitofrontal cortex. In addition, genotype at the Val158Met polymorphism of the catechol- $O$ methyltransferase gene predicts both impulsive choice behavior and activity levels in the APFC and PPC during decision making. These genotype effects remained significant after controlling for alcohol abuse history. These results shed new light on the neurobiological underpinnings of temporal discounting behavior and identify novel behavioral and neural consequences of genetic variation in dopamine metabolism.

Key words: alcoholism; decision making; delay discounting; fMRI; impulsivity; orbitofrontal

\section{Introduction}

Current knowledge regarding the neural basis of temporal discounting derives primarily from lesion studies. For example, damage to the human orbitofrontal cortex (OFC) (Berlin et al., 2004), but not the ventromedial frontal lobe (Fellows and Farah, 2005), increases immediate reward bias, whereas in rats, lesions of the OFC (Mobini et al., 2002; Rudebeck et al., 2006), basolateral amygdala (Winstanley et al., 2004), nucleus accumbens (Cardinal et al., 2001), or hippocampus (Cheung and Cardinal, 2005) bias selection toward immediate rewards. Such selection bias may be viewed as a form of impulsivity (Evenden, 1999). Correspondingly, polymorphisms in several genes in the dopaminergic sys-

Received June 6, 2007; revised Nov. 7, 2007; accepted Nov. 15, 2007.

This work was supported by the Department of Defense and the Wheeler Center for the Neurobiology of Addiction. We thank L. Bergren, L. Altamirano, and B. Inglis for valuable technical assistance.

Correspondence should be addressed to Dr. Charlotte A. Boettiger, Department of Psychology, Davie Hall, CB\#3270, University of North Carolina, Chapel Hill, NC 27599-3270. E-mail: cab@unc.edu.

D01:10.1523/JNEUROSCI.2551-07.2007

Copyright $\odot 2007$ Society for Neuroscience 0270-6474/07/2714383-09\$15.00/0 tem, which targets these brain structures, have been identified as likely contributors to impulsivity (Kreek et al., 2005). Although previous human neuroimaging studies have investigated decision making or the representation of value (Montague et al., 2006), to date only two have directly investigated brain activity associated with deciding between immediate and longer-term payoffs (McClure et al., 2004, 2007). Following economic theories that posit two separate valuation systems to account for the "irrational" discounting of delayed rewards, McClure et al. $(2004,2007)$ identified limbically associated " $\beta$ " regions that are relatively more activated by the availability of a proximate reward and frontoparietal " $\delta$ " regions that are active during all decisions between sooner and later rewards. The $\beta$ and $\delta$ designations refer to the two valuation systems: the $\beta$ system, which is swayed by emotion and overvalues immediate rewards, and the $\delta$ system, which discounts at a constant rate over time (Shefrin and Thaler, 1988; Loewenstein, 1996). However, within behavioral psychology, investigations of temporal discounting have yielded results that do not require competing visceral and rational selection processes to explain immediate reward bias (Rubinstein, 2003; Zauberman 
and Lynch, 2005; Malkoc and Zauberman, 2006; Nussbaum et al., 2006). Rather, alternate theories that address the purely cognitive bases of temporal discounting (Trope and Liberman, 2003; Zauberman and Lynch, 2005) propose that temporal discounting is attributable, at least in part, to how decisions are cognitively framed and that when proximate outcomes dominate conscious thought, more impulsive choice tendencies result. An unanswered question raised by these theories is what brain areas play a role in cognitively mediated bias toward immediate or delayed rewards? To address this question, we used functional magnetic resonance imaging (fMRI) and a modified delay discounting (DD) task (Mitchell et al., 2005, 2007) to identify brain regions associated with immediate reward bias or anti-bias. To maximize the range of individual differences across subjects, participants were either abstinent alcoholics (AA; $n=9$ ) or age-matched controls with no history of substance abuse (CS; $n=10$; see Materials and Methods). In each trial of the task, subjects were instructed to choose between two amounts of money, a smaller amount available "Now" (e.g. "\$80 TODAY") or a larger amount available "Later" (e.g., "\$100 in 1 month") (see Fig. 1).

Results from a previous study, in which we manipulated endogenous opioid levels of subjects engaged in this task, suggested that basal frontal dopamine (DA) levels are inversely related to the frequency of impulsive choices (Mitchell et al., 2007). Moreover, acute elevation of DA reduces immediate reward bias in humans (de Wit et al., 2002). Therefore, in the present study, we assessed genotype at the Val158Met polymorphism of the catechol-O-methyltransferase (COMT) gene, which plays a significant role in regulating frontal DA (Chen et al., 2004). We then tested whether variation at the COMT 158 locus significantly predicted decision-making behavior and/or underlying neural activity.

\section{Materials and Methods}

Subjects. Nineteen healthy, right-handed subjects (eight females; mean age, 28.3 \pm 5.8 ) from the San Francisco Bay Area volunteered and were paid for their participation. Subjects were either self-identified alcoholics that were currently abstinent from alcohol $(n=9)$ or persons with no history of substance abuse $(n=10)$. Five additional subjects were included in the genotyping analysis but were excluded from the fMRI analyses because of excess motion or equipment failure. All subjects had at least a high school education, and the two groups were matched for socioeconomic status, age, intelligence quotient (IQ), and years of education. Alcohol addiction severity (before sobriety for the AA group) was assessed via the Alcohol Use Disorders Identification Test [AUDIT (Saunders et al., 1993)]. Mean AUDIT scores for the AA and CS groups were $19 \pm 7$ and $5 \pm 2$, respectively, with scores $\geq 8$ indicating problem drinking. AA subjects were recruited on the basis of $\geq 2$ weeks of sobriety, and they reported an average 2.5 years of sobriety. Before participation, subjects were screened for medical, neurological, and psychiatric illness and for psychoactive medication. In addition, subjects were urine screened for illicit drug use (Biotechnostix, Markham, Ontario, Canada) and screened for alcohol use via breathalyzer (Lifeloc Technologies, Wheat Ridge, $\mathrm{CO}$ ) before each scanning session. All subjects gave written, informed consent, in accordance with the guidelines of the University of California, Berkeley Committee for the Protection of Human Subjects. Subject payment was not dependent on choice behavior in the task.

Experimental paradigm. Subjects were first provided task instructions and then given a brief practice session to familiarize them with the delay discounting (DD) task. Subjects then performed the DD task within an fMRI scanner, while we obtained a continuous whole-brain bold oxygen level-dependent (BOLD) signal. Scanning was divided into eight functional scans of 37 trials each ( 32 choice trials, and 5 "null" trials), in which trial types were presented in a pseudorandom order that was unique for each subject (total scanning duration of $\sim 1 \mathrm{~h}$ ). At the start of each trial,

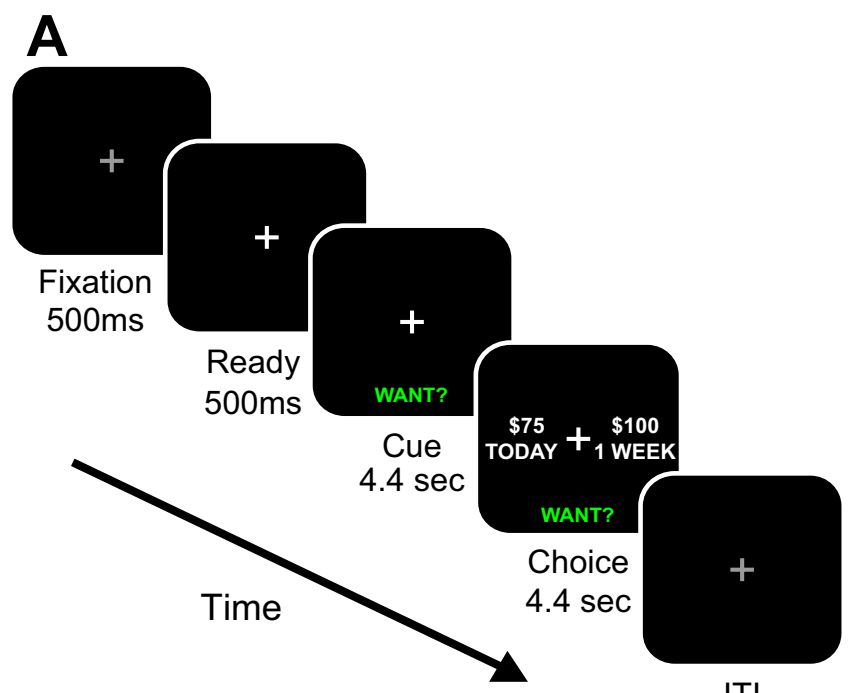

ITI
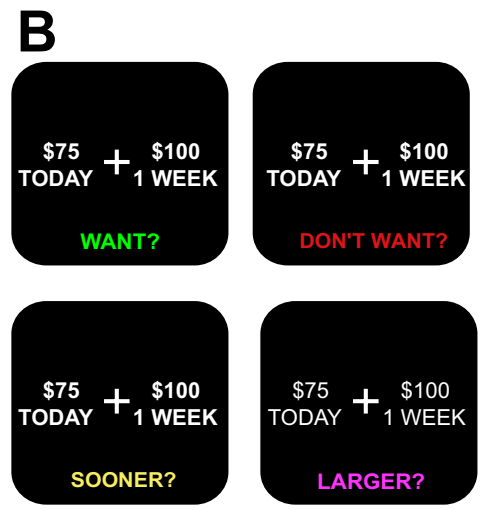

4.4-8.8 sec

Figure 1. Illustration of behavioral paradigm. $A$, The temporal sequence of events are shown for one example W trial. Illumination of a fixation point (Ready) indicated the initiation of each trial. The instruction cue was then displayed for $4.4 \mathrm{~s}$, alerting the subject to the upcoming trial type. The two options (Now and Later) were then presented while the instruction cue remained on the screen. The specific dollar amounts (from $\$ 1$ to $\$ 100$ ) and the time of availability (from the day of the experiment to 6 months later) varied across trials, as did the differences between each option. The choices remained on the screen for $4.4 \mathrm{~s}$; however, subjects had a total of $6.6 \mathrm{~s}$ to indicate their choice after the appearance of the two options. ITI, Intertrial interval. B, Depiction of the four trial types. The appearance of each option pair was preceded by a cue that indicated trial type, instructing the subject how to select between the Now and Later options. For W trials, subjects were to select the option they preferred. The SOONER (S) and LARGER (L) trials served to control for activity that reflected objective comparison of the two options, rather than subjective preference; these are considered together as $\mathrm{CON}$ trials. To assess the ability of the subjects to control unintended motor responses, we included trial type DW, in which subjects were instructed to choose the option they preferred and then press the button corresponding to the opposite choice.

subjects were shown a cue instructing them how to select between the two subsequently displayed hypothetical reward options, each consisting of a dollar amount at a point in time (Fig. 1). We refer to these two alternatives as Now and Later. The Later option was one of six "full" amounts $(\$ 1, \$ 2, \$ 5, \$ 10, \$ 20$, or $\$ 100$ ) at one of five future delays (1 week, 2 weeks, 1 month, 3 months, or 6 months). The Now option was a lesser amount available "TODAY." The percentage difference between the two amounts was randomly selected from the set $[30 \%, 15 \%, 10 \%, 5 \%]$. The right-left position of the Now and Later options was randomized across trials. Subjects indicated their choice by pressing one of two buttons on an MRI-compatible keypad. Instruction cues were one of four types: WANT (W), DON'T WANT (DW), SOONER, and LARGER, the latter two being considered together as CONTROL $(\mathrm{CON})$ (Fig. $1 \mathrm{~B}$ ). In the W condition, subjects chose the option they preferred. In the DW condition, subjects were asked to make the same evaluation but to press the 
button corresponding to the opposite choice. On CON trials, subjects selected the side with either the sooner time point or larger amount of money, depending on the instruction cue. Performance on CON trials verified that subjects comprehended the task and were compliant with task instructions. As described in detail below, the DW condition allows for a gross assessment of motor impulsiveness, by comparing inferred choices from the DW trials as a function of time with actual choices in the $\mathrm{W}$ condition as a function of time. This procedure serves to rule out spurious impulsive choice as a result of unintended motor responses. Trial types were pseudorandomly ordered, with weighted ratios of $1: 2$ for the $\mathrm{W}$ condition and 1:6 for each of the other conditions (Fig. 1). The order of intertrial intervals did not vary across subjects; however, the trial type, Later amount, Later time, and discount rate were pseudorandomly selected on each trial. Because the choice condition followed the cue condition at a fixed delay for each trial, we included null trials (five trials in each functional scanning run), in which the instruction cue appeared but no options followed. This trial procedure meant that subjects saw each of the 120 possible choices two or three times, although not necessarily within the same trial type.

Analysis of behavioral data. Behavioral data were analyzed using Microsoft (Redmond, WA) Excel and/or SPSS (Chicago, IL) software. The proportion of Now choices relative to all $\mathrm{W}$ condition choices made was calculated for each subject; this quantity, referred to as the impulsive choice ratio (ICR), ranged from 0 (all Later) to 1 (all Now). A number of other indices of impulsive choice were also calculated, as described previously (Mitchell et al., 2005), yielding qualitatively similar results (data not shown). The task design does not allow robust determination of a hyperbolic discount rate $(k)$, thus $k$ was not used as a dependent measure. Reward preference in DW trials was inferred to be the nonselected option. On the basis of these DW responses, we calculated an inferred ICR (iICR) for each delay time. Subjects demonstrated a significant effect of delay time on ICR (repeated-measures ANOVA, $p<0.001$ ), with no significant interaction between delay and group effects $(p=0.772)$, consistent with our previous results (Mitchell et al., 2005, 2007). This fact allowed us to calculate the absolute difference between the ICR and IICR for each delay time and use the sum of $\mid$ ICR - iICR $\mid$ across delay times as a gross index of motor error.

For single-factor statistical comparisons between subject groups, we used unpaired two-tailed $t$ tests for continuous measures and $\chi^{2}$ tests for categorical measures. For multifactorial comparisons, we used mixeddesign ANOVA, with group (AA vs CS) as a between-subjects factor. To ensure the validity of parametric statistical tests, we applied an arcsineroot transformation to ICR data before making statistical comparisons. Analyses of covariance (ANCOVA) were used to assess the role of the COMT genotype, after controlling for group effects (AA vs CS).

Image acquisition and analysis. T2*-weighted echo planar images (EPIs) were acquired on a Varian/Inova (Palo Alto, CA) whole-body 4T magnetic resonance scanner. A gradient echo, echo planar sequence (repetition time, $2200 \mathrm{~ms}$; echo time, $28 \mathrm{~ms}$; flip angle, $20^{\circ}$ ) was used to detect BOLD contrast. The sequence used one-shot k-space acquisition with a phase map correction to reduce Nyquist ghosts. Coverage of the ventral/ orbital prefrontal cortex was facilitated by orienting the phase-encoding axis along the rostrocaudal image dimension and sampling $\mathrm{k}$-space in the order positive to negative (De Panfilis and Schwarzbauer, 2005). This served to minimize the effects of EPI phase-encoding distortion and expanded rather than compressed the remaining distortion. Wholebrain coverage was obtained by acquiring $403.5 \mathrm{~mm}$ coronal slices with a $0.5 \mathrm{~mm}$ interslice gap $\left(64 \times 64\right.$ matrix; field of view, $\left.22.4 \mathrm{~cm}^{3}\right)$, yielding an in-plane resolution of $3.5 \mathrm{~mm}$. Each $\mathrm{fMRI}$ acquisition was preceded by $20 \mathrm{~s}$ of dummy gradient radiofrequency pulses, to achieve steady-state tissue magnetization and to minimize startle-induced motion in the functional data. Coplanar T1-weighted gradient echo multislice anatomical images (GEMs) were acquired for each participant. In addition, an axial magnetization-prepared fast low-angle shot high-resolution (MPFLASH) T1-weighted image was acquired for use in spatial normalization. E-Prime software (PST, Pittsburgh, PA) synchronized the stimulus display to the fMRI acquisition and recorded subject responses via an MRI-compatible fiber-optic keypad. A liquid crystal display projector (Epson, Long Beach, CA) projected stimuli onto a backlit projection screen (Stewart, Torrance, CA) within the magnet bore, which the subjects viewed via a mirror mounted within the head coil.

fMRI data were processed off-line using a combination of in-house software and the statistical parametric software SPM2 (Wellcome Department of Imaging Neuroscience, London, UK; www.fil.ion.ucl.ac.uk/ spm). First, images were reconstructed into Cartesian space and sinc interpolated in time to correct for differences in slice-time acquisition. Data were then motion corrected using a six-parameter, rigid-body, least-squares algorithm (Friston et al., 1995), realigning all volumes to the first EPI volume, and smoothed with a $7 \mathrm{~mm}$ full-width halfmaximum Gaussian smoothing kernel. Each subject's coplanar (GEMs) and high-resolution (MPFLASH) anatomical images were coregistered to the first EPI volume, normalized to Montreal Neurological Institute (MNI) atlas space, and resampled to $2 \mathrm{~mm}^{3}$ isotropic voxels. The resulting normalization parameters were subsequently applied to the statistical contrast images (see below).

The functional data were analyzed in an event-related manner within the framework of the modified general linear model using SPM2. Each subject's model included a design matrix with regressors of interest consisting of $\delta$ functions corresponding to the onset time of each experimental event type (ready, instruction cue, choice pair) convolved with a canonical hemodynamic response function. Data were high-pass filtered with a cutoff of $128 \mathrm{~s}$ and corrected for serial autocorrelations with a restricted maximum likelihood algorithm using a second-order autoregressive model (AR2 plus white noise). Linear contrast images for each subject were calculated from the regressor parameter estimates. The contrast images were then normalized into MNI space using the parameters derived from the coregistered MPFLASH.

The main goal of our fMRI analysis was to determine which brain regions show activity that predicts individual immediate reward bias during subjective preference-based decision making. These were identified by first identifying brain areas that were differentially active during subjective choice relative to objective choice trials (i.e., $\mathrm{W}-\mathrm{CON}$ ). The $\mathrm{W}$ and $\mathrm{CON}$ conditions are equivalent in terms of stimulus properties and motor response. This contrast can also be construed as identifying areas that are differentially active during deliberative or effortful decision making relative to more rapid responding. A simple correlation analysis was then performed that identified voxels in which activity in the choice contrast $(\mathrm{W}-\mathrm{CON})$ directly correlated with ICR across subjects. Significant positive correlations were interpreted as an association with Now preference, whereas significant negative correlations were interpreted as an association with Later preference. To allow direct comparison with previously published studies (McClure et al., 2004, 2007; De Martino et al., 2006), we report results significant at $p<0.001(t=3.65)$, uncorrected for multiple comparisons, with a minimum cluster size of five contiguous voxels. To control for multiple comparisons, we confirmed that the reported sites all survived voxelwise correction for familywise type I error ( $\alpha<0.05$; whole-brain volume), determined by randomly permuting the ICR values and the $(\mathrm{W}-\mathrm{CON})$ contrast parameter estimates in 8000 new analyses (Nichols and Holmes, 2002; Nichols and Hayasaka, 2003) using the SnPM3 analysis package (http:// www.sph.umich.edu/ni-stat/SnPM/). For display purposes, statistic images are shown at a threshold of $p<0.005$ (uncorrected).

Genetic analysis. DNA extraction and analysis was conducted using standard methods on samples obtained from all subjects who gave informed consent $(n=25)$. The Ernest Gallo Clinic and Research Center Genomics Core performed genotyping of the Val108/158Met polymorphism of the COMT gene with PCR using TaqMan technology (Applied Biosystems, Foster City, CA). Seven subjects were homozygous for the $158^{\mathrm{Val}}$ allele, and eight subjects were homozygous for the $158^{\mathrm{Met}}$ allele of the COMT gene; 10 heterozygote subjects carried one $158^{\mathrm{Val}}$ and one $158^{\mathrm{Met}}$ allele of the COMT gene; genotype groups were not significantly different with respect to age, education, socioeconomic status, IQ, gender, or alcohol abuse.

\section{Results}

\section{Behavioral data}

Consistent with our previous findings (Mitchell et al., 2005, 2007), the ratio of Now choices (ICR) was significantly higher in 

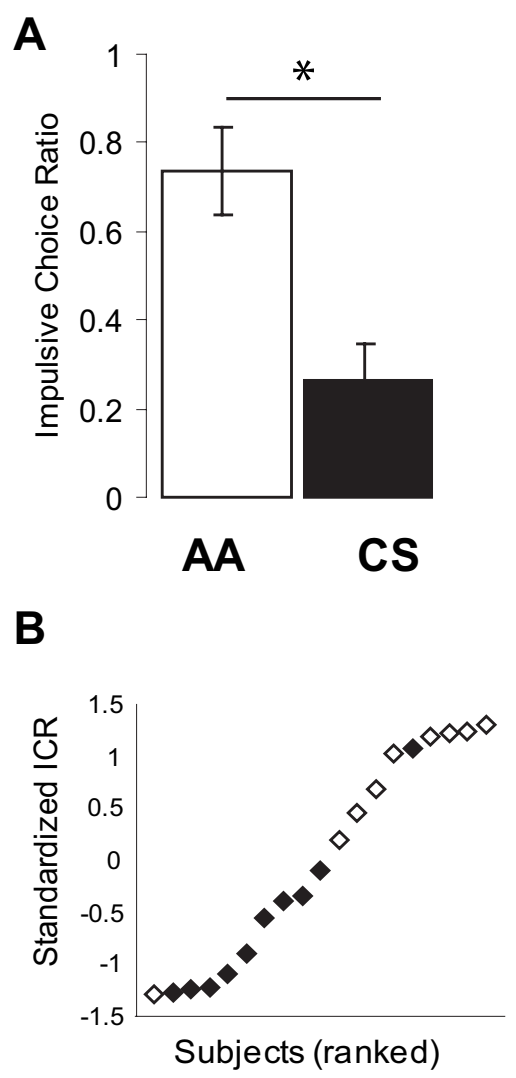

Figure 2. Behavioral results. $\boldsymbol{A}$, Ratio of impulsive (Now) choices as a function of subject group. The AA group made significantly more impulsive choices $\left(0.74>0.26\right.$; ${ }^{*} p=0.002$; $\left.t_{(17)}=3.56\right)$ than the $(S$ group. Error bars denote SEM. A value of 0 represents always choosing Later over Now, whereas a value of 1 represents the converse. $\boldsymbol{B}$, Standardized ICR for each subject in rank order. Units are in SDs, with mean equal to $0 . \diamond, A A ; \diamond, C S$.

the AA group than in the CS group (Fig. $2 A$ ). The group difference in ICR was not attributable to a failure to deliberate among the AA group, because the average reaction times (RTs) did not differ between groups (AA, $2187 \pm 142 \mathrm{~ms}$; CS, $2146 \pm 177 \mathrm{~ms}$; $p=0.88$ ). Nor was the ICR difference attributable to greater motor error in the AA group, because an index of motor error did not differentiate the groups; in fact, we saw a slight trend toward more motor error in the CS group (AA, 0.47 ; CS, $0.67 ; p=0.15$ ). A repeated-measures ANOVA comparing choices in the $\mathrm{W}$ and DW conditions across delay times demonstrated no significant difference between ICRs calculated from the two conditions ( $p=$ $0.924)$, indicating minimal motor error. In addition, we did not detect a significant interaction between trial type (W vs DW) and group, although again we saw here a trend toward greater mismatch in the CS group $(p=0.147)$. The AA and CS groups performed equivalently on the CON trials, both in terms of accuracy (AA, $96 \pm 1 \%$; CS, $98 \pm 1 \% ; p=0.59)$ and RT (AA, $1750 \pm 130 \mathrm{~ms} ; \mathrm{CS}, 1558 \pm 186 \mathrm{~ms} ; p=0.38)$. We note that the CON trial RTs were significantly faster than the W trial RTs $\left(F_{(1,18)}=35.4 ; p<0.001\right)$, consistent with the expectation that the (deliberative) $\mathrm{W}$ condition uses processes in addition to those used during objective criteria-based decision making. We observed no significant group by trial type effect on $\mathrm{RT}\left(F_{(1,18)}<1\right.$; $p=0.39$ ). Although the ICR group means were significantly different, each group spanned a range of impulsiveness (Fig. 2B).

\section{Neural correlates of Now versus Later preference}

To localize brain areas that were more active during subjective decision making, we first generated contrast images $(\mathrm{W}-\mathrm{CON})$
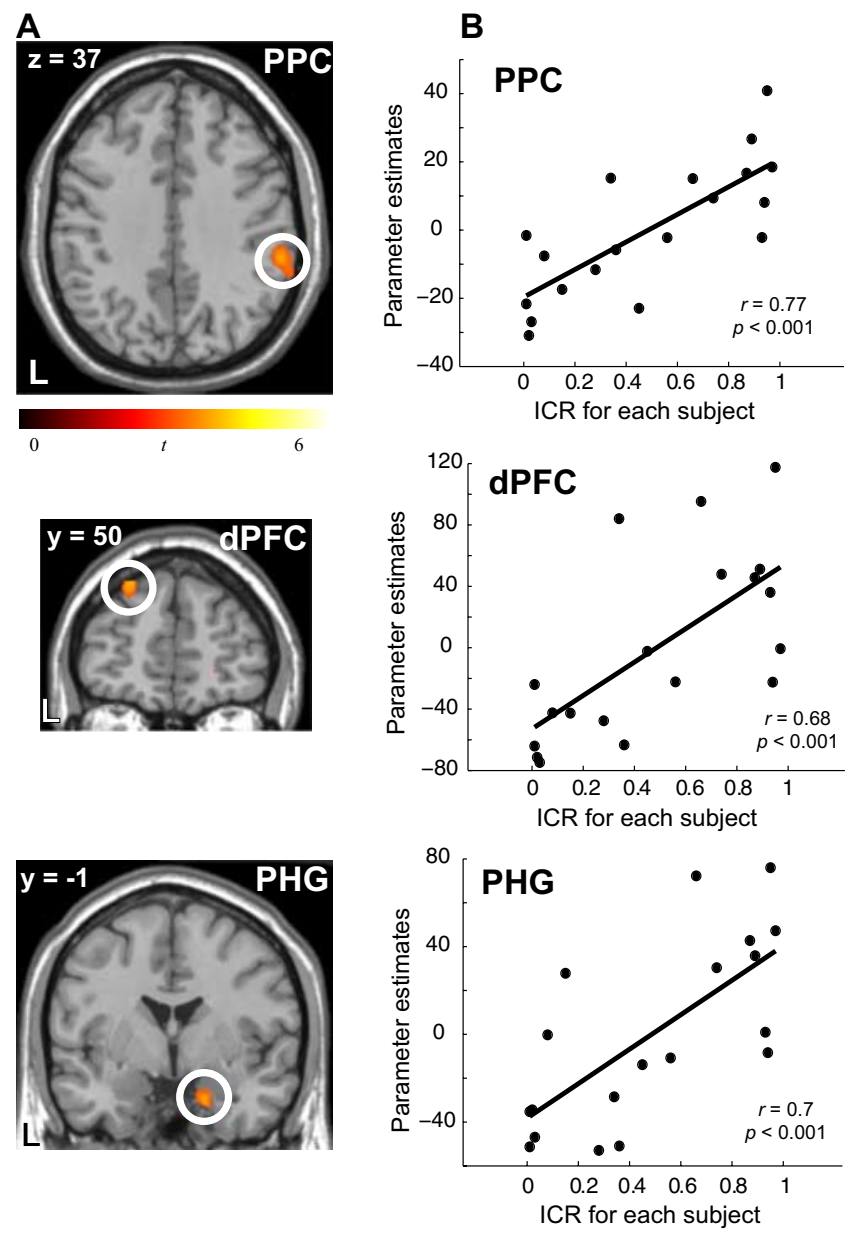

Figure 3. Selection of impulsive choice across subjects: fMRI correlation analysis. Brain regions showing a significant positive correlation between activity during subjective Now versus Later decision making and ICR. Brain activity indices derived from the subjective choice contrast (W - CON); see Materials and Methods for greater detail. $\boldsymbol{A}$, MNI space coordinates $(x, y, z)$ and $t$ values for the PPC (top; 66, $-42,44 ; t=5.03$ ), dPFC (middle; $-22,50,44 ; t=3.79$ ), and PHG (bottom; 18, $-4,-28 ; t=4)$ ). Criterion threshold was $p<0.001$ (uncorrected), with a minimum cluster size of five contiguous voxels; results are shown at $p<0.005$ for display purposes. These data also survived permutation-based voxelwise correction of familywise error $(p<0.05)$. L, Left. $\boldsymbol{B}$, Corresponding correlation plots for each subject's contrast parameter estimate (an index of relative activity) versus ICR for the peak of each region identified at left; the peak regression statistic is displayed in each plot.

identifying brain areas that were relatively more active when subjects were deciding whether they preferred a smaller, immediate reward (Now) or a larger, delayed reward (Later) than when they were simply determining which option was Now or Later and selecting it. Because our main interest was not in identifying decision-making regions per se but in identifying areas that may bias choice toward or away from immediate rewards, we consequently entered the choice ( $\mathrm{W}-\mathrm{CON}$ ) contrasts from each subject into a mapwise simple regression analysis. This correlation analysis exploited the intersubject variability in ICR (Fig. $2 B$ ) to identify differences in the BOLD signal associated with the individual tendency to choose Now over Later. This approach obviated potential problems associated with simply comparing the AA and CS groups (Pfefferbaum et al., 1997). Using the ICR of each subject as a between-subjects statistical regressor, we found a significant positive correlation between ICR and activity during preference-based decision making in the dorsal prefrontal cortex (dPFC), the posterior parietal cortex (PPC), and the anterior 
Table 1. Brain areas demonstrating a significant positive correlation between ICR and the subjective choice contrast image (W - CON)

\begin{tabular}{llll}
\hline Brain region & Hemisphere & MNI coordinates $[x, y, z$ (in mm) & $t$ value \\
\hline Supramarginal gyrus (parietal lobe) & Right & $66,-42,44$ & 5.03 \\
Cerebellum, posterior lobe (lobule VIII) & Right & $36,-52,-62$ & 4.63 \\
Middle temporal gyrus & Right & $56,-52,-2$ & 4.46 \\
Inferior temporal gyrus & Right & $68,-50,-12$ & 4.24 \\
Parahippocampal gyrus (gyrus ambiens, entorhinal & & & 4.00 \\
$\quad$ area, semilunar gyrus) & Right & $18,-4,-28$ & 3.79 \\
Superior frontal gyrus & Left & $-22,50,44$ &
\end{tabular}

Activated anatomical region, hemisphere, coordinates of the local maxima of significance based on the MNI coordinate system, and the peak $t$ value are given. The reported regions survived a threshold of $p<0.001$ (uncorrected; 5 voxel minimum), as well as a permutation-based voxelwise correction of familywise type l error $(p<0.05)$.
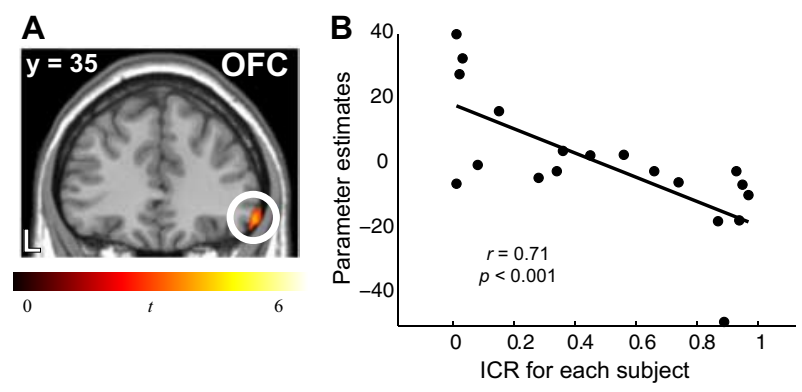

Figure 4. Suppression of impulsive choice across subjects: fMRI correlation analysis. The sole brain region showing a significant negative correlation between activity during subjective Now versus Later decision making and ICR. Methods and conventions are as for Figure 3. $\boldsymbol{A}$, Right lateral OFC $(8,36,-10 ; t=4.13)$. L, Left. $\boldsymbol{B}$, Plot of each subject's OFC Now versus Later subjective decision-making parameter estimate as a function of ICR for the peak of the region displayed at left; the peak regression statistic displayed in plot.

parahippocampal gyrus (PHG) (Fig. 3; see Table 1 for all sites identified). The peak of the PHG locus is medially adjacent to the amygdala, and its extent overlaps with an amygdalar region reported to play a role in emotion-based, as opposed to analytic, decision making (De Martino et al., 2006). In summary, in these brain areas, activity associated with deciding whether to choose Now or Later was highest in the subjects who were least willing to wait. In contrast, we observed a significant negative correlation of ICR with activity during subjective decision making in the right lateral OFC (Fig. 4, Table 2). That is, we observed the greatest activity in the OFC of subjects most likely to select the larger, delayed reward.

\section{Decision-making activity in the Now and Later region of interest varies with ICR independent of group}

The results of two further analyses strengthen the conclusion that the correlations between BOLD signal during decision making and individual ICR described above reflect true brain-behavior relationships rather than group differences consequent to alcohol abuse. First, for each region of interest (ROI), we used multiple regression analysis to test whether ICR retained a significant prediction of BOLD signal during decision making after controlling for any effect of group. In each ROI, we found that a significant correlation between ICR and BOLD signal remained after controlling for group [standardized coefficients: dPFC, 0.651 ( $p=$ 0.018 ); PPC, 0.628 ( $p=0.011)$; PHG, 0.813 ( $p=0.003)$; OFC, $-0.845(p=0.002)]$. Second, we tested for a significant correlation between ICR and BOLD signal in the CS group alone. Again, we found significant correlations in each ROI between ICR and BOLD signal [standardized coefficients: dPFC, $0.704(p=$ 0.023); PPC, 0.651 ( $p=0.042)$; PHG, 0.657 ( $p=0.039)$; OFC, $-0.916(p<0.001)]$. Together, these results indicate that our findings are not simply a result of group differences in alcohol abuse history.

\section{Comparing Now versus Later circuitry in alcoholics and controls}

Although our analysis demonstrates that activity within the Now and Later ROI reliably correlates with temporal discounting behavior, independent of alcohol abuse history, if the relative activity in the Now and Later regions distinguish AA from CS, the potential clinical utility of our findings would be enhanced. Direct comparison of the normalized decisionmaking activity in Now and Later ROI confirmed that activity differences in these ROIs differed significantly between the two groups (Later-Now: CS, 10.0; AA, $-46.5 ; p=0.02 ; t_{(17)}=2.49$ ).

\section{Main effect of COMT genotype on frequency of impulsive choices}

A two-way factorial ANOVA with ICR as the dependent variable confirmed a main effect of group $\left(F_{(1,19)}=12.123 ; p=0.002\right)$ (Fig. $2 A$ ) and also demonstrated a main effect of COMT genotype $\left(F_{(2,19)}=5.693 ; p=0.012\right)$ (Fig. 5). There was no significant group by genotype interaction $(p>0.3)$. A post hoc contrast indicated that the genotype effect was attributable to a significant difference between the $158^{\mathrm{Val} / \mathrm{Val}}$ genotype and the other two genotypes ( $p=0.009)$; the $158^{\mathrm{Val} / \mathrm{Met}}$ and $158^{\mathrm{Met} / \mathrm{Met}}$ groups did not differ significantly from one another $(p=0.126)$. The significant effect of genotype remained after controlling for the effects of group (ANCOVA; $\left.F_{(2,21)}=5.181 ; p=0.015\right)$, indicating that the genotype effects on decision making are independent of alcohol abuse history. Distribution of AA subjects was not significantly different across genotypes: $158^{\mathrm{Val} / \mathrm{Val}}, 4$ of $7 ; 158^{\mathrm{Val} / \mathrm{Met}}, 5$ of $10 ; 158^{\mathrm{Met} / \mathrm{Met}}, 3$ of $8\left(\chi^{2}=1.3 ; p=0.73\right)$.

\section{Main effect of COMT genotype on fronto-parietal activity during decision making}

To test whether the COMT genotype also had an effect on BOLD signal during subjective decision making in the distributed network of brain regions identified based on robust prediction of ICR (Figs. 3, 4), we performed a two-way factorial ANOVA (genotype by group) with $(\mathrm{W}-\mathrm{CON})$ contrast parameter estimate as the dependent variable. A significant effect of COMT genotype on decision-making activity was found within two of the predefined ROIs: the $\operatorname{dPFC}\left(F_{(2,13)}=3.869 ; p=0.048\right)$ (Fig. 6, left) and the $\operatorname{PPC}\left(F_{(2,13)}=5.901 ; p=0.015\right)$ (Fig. 6, right). These genotype effects remained significant after controlling for effects of group using ANCOVA (dPFC: $F_{(2,15)}=3.834, p=0.045$; PPC: $F_{(2,15)}=5.911, p=0.013$ ). We found no significant genotype by group interaction effect in either ROI ( $p>0.6$ for both). No significant effect of genotype on brain activity was seen in the other ROI after controlling for effects of group. These genotype effects were driven by differences between $158^{\mathrm{Val} / \mathrm{Val}}$ and $158^{\mathrm{Val} / \mathrm{Met}}$ genotypes in both the $\operatorname{dPFC}(p=0.026)$ and the PPC $(p=0.005)$. Although our results argue against a dose effect of the $158^{\mathrm{Val}}$ allele, our sample size precludes ruling out such an effect.

\section{Discussion}

Deciding for the future

Evaluating the costs and benefits of different behavioral options and predicting which action is optimal is a critical function of the 
CNS. In evaluating alternative actions, humans have a great capacity to integrate outcomes across multiple timescales. For example, we can overlook the short-term deprivation of not spending money now to reap the long-term benefits of compound interest. However, the capacity to make optimal decisions based on the integration of outcomes at multiple timescales matures during development and ultimately varies greatly across the adult population. Moreover, a number of neurobehavioral disorders, including alcoholism, are characterized by a tendency to overestimate short-term costs and benefits and underestimate long-term outcomes. A better understanding of the neurobiological basis of immediate reward bias during decision making may thus lead to improved evaluation and treatment of such disorders, which would have broad social impact. The present results represent a significant step toward this goal.

Our data build on previous investigations of decision making, by identifying brain areas in which activity during decision making predicts individual preference for Now versus Later. Each of the regions we identified has been experimentally linked to the representation of value or outcome comparison in human and/or animal studies; however, our data extend previous work by providing additional insight into the different roles each brain region may play in the process of choosing between immediate and delayed rewards. Furthermore, we have determined that a functional variation in COMT genotype influences impulsive decision making, an intermediate phenotype for use in studying the neural bases of addiction (Reynolds, 2006), and its underlying fronto-parietal activity.

\section{Frontal hyperactivity and immediate reward bias}

The rostral dPFC is active when humans decide between sooner or later rewards (McClure et al., 2004, 2007). In addition, activity in the dPFC is linked experimentally to representations of the reward value of potential actions and the outcomes of past choices during decision making in monkeys (Barraclough et al., 2004). In humans, activity in the left dPFC is also associated with "reward bias," an enhanced tendency to respond to rewardrelated cues (Pizzagalli et al., 2005). Together with our present dPFC findings, these data suggest that heightened left dPFC activity during decision making biases selection toward the greatest short-term benefit. In our study, the immediate outcomes are something or nothing, thus in the short-term, the Now option is greatest.

Deciding between Now and Later in our task may also require sustained information maintenance and manipulation in dPFC circuits (D'Esposito, 2007). For example, to calculate accrued interest during the delay for the immediate reward, a subject needs to hold in working memory the Now amount, the interest rate, and the duration of the delay to calculate the future Now value, which can then be compared with the Later amount to make a choice. We have previously found that AA subjects have an interest rate criterion threshold threefold higher than do CS subjects (Mitchell et al., 2007), a disparity that could result from inefficient calculation and comparison. However, the inefficiency hypothesis conflicts with our interpretation of the OFC activity seen in this study. Thus, an alternative explanation is that identified Now regions may represent proximate outcome comparisons, whereas activity in the lateral OFC represents more temporally distal outcome comparisons, with the relative

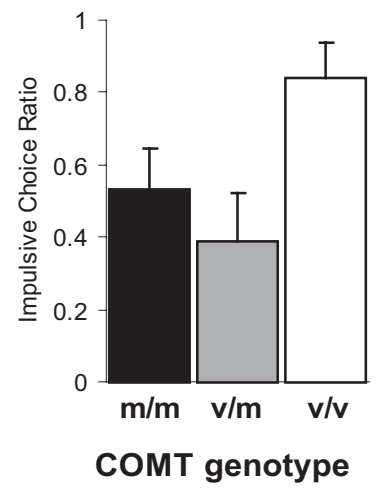

Figure 5. Genotype at the COMT 158 locus predicts impulsive choice behavior. The ratio of impulsive choices as a function of genotype is shown. There was a significant main effect of genotype at the COMT 158 locus on impulsive choice probability, even after controlling for the effects attributable to group (AA vs CS; ANCOVA, $F_{(2,21)}=5.181, p=0.015$ ). The $158^{\mathrm{Val} / \mathrm{Val}}$ genotype was associated with significantly more frequent selections of Now over Later than were the other two genotypes ( $p=0.012$ ), which did not significantly differ from one another $(p=0.144)$. Error bars denote SEM. m/m, $158^{\text {Met/Met }} ; \mathrm{v} / \mathrm{m}, 158^{\text {Val } / M e t} ; \mathrm{v} / \mathrm{v}, 158^{\text {Val } / \text { Val }}$.
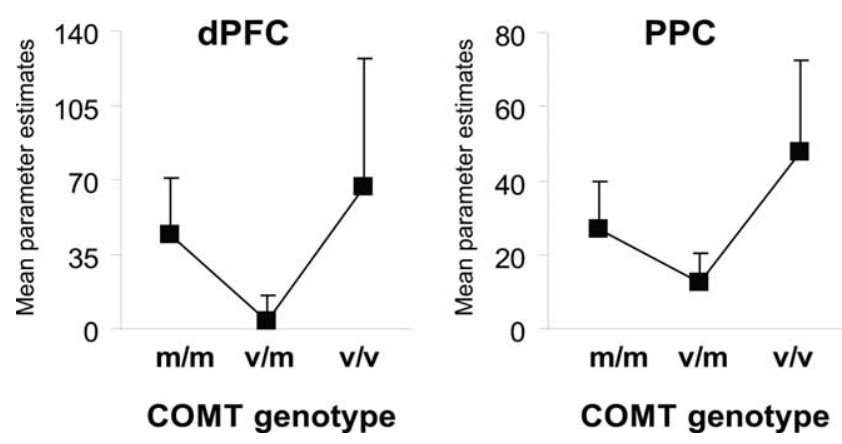

Figure 6. COMT $158^{\mathrm{Val} / \mathrm{Val}}$ genotype predicts hyperactivation in the $\mathrm{APFC}$ and PPC during decision making. Parameter estimates indexing brain activity during Now versus Later subjective decision making as a function of the COMT Val158Met genotype within the $\mathrm{APFC}$ and PPC $\mathrm{ROI}$ (averaged across voxels). There was a significant main effect of genotype at the COMT 158 locus on BOLD signal in both regions, even after controlling for subject group effects (ANCOVA; $\left.\mathrm{dPFC}, F_{(2,15)}=3.834, p=0.045 ; \mathrm{PPC}: F_{(2,15)}=5.911, p=0.013\right)$. The $158^{\mathrm{Val} / \mathrm{Val}}(\mathrm{v} / \mathrm{v})$ genotype was associated with significantly greater activation in the $\mathrm{APFC}$ and $\mathrm{PPC}$ during subjective decision making ( $\mathrm{dPFC}, p=0.026 ; \mathrm{PPC}, p=0.005$ ). Error bars denote SEM. $\mathrm{m} / \mathrm{m}, 158^{\mathrm{Met} / \mathrm{Met}}$; $\mathrm{v} / \mathrm{m}, 158^{\mathrm{Val} / \mathrm{Met}} ; \mathrm{v} / \mathrm{v}, 158^{\mathrm{Val} / \mathrm{Val}}$.

strength of immediate versus long-term choice outcomes predicting an individual's selection bias.

\section{Value and the parietal lobe}

Like the $\mathrm{dPFC}$, the PPC demonstrated activity during decision making that scales with immediate reward bias. This is consistent with the observation of McClure et al. $(2004,2007)$ that this area is active during decisions between sooner and later rewards, and with data linking the PPC more generally to numerical distance calculation (Pinel et al., 2001). Moreover, in monkeys, the relative subjective value of competing alternative actions is represented in PPC neural activity (Dorris and Glimcher, 2004; Sugrue 
et al., 2004). These electrophysiology data suggest that value computation per se does not occur in the PPC, but rather that the PPC is where the abstract relative values of available options are compared and mapped on to motor responses (Sugrue et al., 2004). The fact that we saw higher PPC activity in subjects inclined to select immediate rewards suggests a few possible explanations, first that Now-preferring subjects perceived a greater "distance" between the two options, which translated to enhanced activity in the PPC. Alternatively, as proposed for the dPFC, higher activity may reflect processing inefficiency in this region. Given that a similar pattern was seen in the $\mathrm{dPFC}$, these areas may form part of a functional circuit that cooperates in the process of numerical comparisons that inform subjective choice.

\section{Now versus Later preference: a role for the rostomedial temporal lobe region}

An area not previously identified as playing a role in Now versus Later decision making is the rostromedial temporal lobe. However, our finding that rostral PHG activity during decision making correlates with immediate reward bias is consistent with the fact that during another type of decision making, activity in an adjacent and overlapping site in the amygdala scales with individual susceptibility to counterfactual effects on decisions (De Martino et al., 2006). Our finding is also consistent with lesion data indicating that the amygdala mediates the role of affect on decision making (Baxter and Murray, 2002; Hsu et al., 2005; Balleine and Killcross, 2006), possibly by representing the affective value of anticipated outcomes (Kahn et al., 2002; Yacubian et al., 2006). Our PHG findings thus suggest greater negative anticipation (or cost) of waiting for a reward in those preferring Now. If true, impulsive decision making may be attributable not only to devaluation of temporally distant rewards but also to heightened negative expectations of waiting for outcomes, an explanation not yet explored experimentally.

\section{Lateral OFC and choosing to wait for bigger payoffs}

In contrast to the above Now-favoring areas, higher activity during decision making in the right lateral OFC correlated directly with the inclination to wait for larger gains. This is consistent with OFC lesions promoting impulsive choice (Mobini et al., 2002; Rudebeck et al., 2006) and with OFC neurons playing a role in value-based choice (Izquierdo et al., 2004; Roesch and Olson, 2004; Schoenbaum and Roesch, 2005; Padoa-Schioppa and Assad, 2006). It is also consistent with lateral OFC activity representing relative monetary reward value (Elliott et al., 2003). We speculate that the OFC preferentially represents more distal outcome comparisons, or the integration of relative outcome comparisons across multiple timescales, possibilities not yet addressed in either humans or animals.

Our finding of reduced OFC activity during decision making among AA subjects is consistent with the common finding of OFC dysfunction in substance abusers (London et al., 2000; Volkow and Fowler, 2000; Dom et al., 2005). Moreover, our data indicate a specific dysfunction of the lateral OFC that may contribute to the persistence of addictive disorders. Abnormal activity in the same OFC site was observed in a positron emission tomography study of abstinent opiate and methamphetamine abusers performing a gambling task (Ersche et al., 2005), suggesting that abnormal activity in the lateral OFC when deciding between rewards may be a reliable addiction biomarker. Therefore, determining whether this biomarker is an addiction vulnerability or relapse risk factor are important future questions.

\section{Ventral striatum: reward sensitivity, not behavioral choice?}

We note that our analyses did not identify the ventral striatum (VS), in apparent contrast to the recent finding of Hariri et al. (2006) that VS responses to proximate reward stimuli predict individual temporal discounting rates measured in a nonscanning context. However, we also note that our analyses instead identified brain areas showing activity during decision making that predicts Now versus Later choices when those options are relatively similar. Moreover, our task design controlled for effects attributable to stimulus reward properties, including magnitude and temporal proximity. Finally, we note that recent data from McClure et al. (2007) show that VS activity during decision making is linked to stimulus properties but not to choice behavior. Thus, the fMRI signal in the human VS appears to represent individual reward sensitivity (Knutson et al., 2005), rather than reward comparison or choice.

\section{COMT: cortical DA and temporal discounting}

Our results indicate that genetic variation in COMT function influences decision-making behavior and underlying activity in brain regions associated with immediate reward preference. Specifically, individuals carrying the enzymatically less active $158^{\text {Met }}$ allele of the COMT gene exhibit decreased immediate reward bias and show less fronto-parietal activity during decision making compared with those homozygous for the $158^{\mathrm{Val}}$ allele. Such genetically driven variation in COMT function and subsequent fronto-parietal function may also contribute to other forms of impulsive behavior (Cools and Robbins, 2004; Congdon and Canli, 2005; Kreek et al., 2005). Studies linking COMT to alcoholism have been equivocal (Ishiguro et al., 1999; Kauhanen et al., 2000; Kim et al., 2006; Samochowiec et al., 2006; Sery et al., 2006), although some data suggest that alcoholic subphenotypes may associate with specific COMT diplotypes (Tiihonen et al., 1999; Wang et al., 2001; Kweon et al., 2005; Enoch et al., 2006). We did not find a significantly skewed distribution of AA subjects across COMT genotypes; however, the possibility remains that the $158^{\mathrm{Val} / \mathrm{Val}} \mathrm{COMT}$ genotype may confer risk for an impulsive alcoholism subtype.

The COMT $158^{\mathrm{Val} / \mathrm{Val}}$ genotype is also associated with poorer performance on frontal-dependent tasks and greater frontal activation relative to subjects with at least one $158^{\text {Met }}$ allele (Egan et al., 2001; Blasi et al., 2005; Minzenberg et al., 2006; Winterer et al., $2006 a, b)$. Such results have been interpreted as processing inefficiency in frontal circuitry for the $158^{\mathrm{Val} / \mathrm{Val}}$ genotype (Tunbridge et al., 2006), particularly during tasks that require maintenance of stable representations (Bilder et al., 2004; Nolan et al., 2004; Tunbridge et al., 2006). However, the role of the COMT 158 genotype in frontal function is not without controversy. In patients with Parkinson's syndrome, the $158^{\mathrm{Val} / \mathrm{Val}}$ genotype predicts increased BOLD signal in the PFC and PPC and is associated with better performance in a planning task (Williams-Gray et al., 2007). The relationship between COMT effects on BOLD and frontal task performance may reflect task-dependent sensitivity to frontal DA (Nolan et al., 2004) and the inverted U-shaped relationship between working memory performance and frontal DA (GoldmanRakic et al., 2000).

A COMT 158 genotype by disease interaction is seen in Parkinson's disease (Williams-Gray et al., 2007), but not in schizophrenia (Egan et al., 2001), for BOLD and frontal-dependent tasks. Moreover, Parkinson's disease is characterized by selective (at least initially) striatal DA depletion (Kish et al., 1988), whereas schizophrenia is thought to reflect relative striatal hyperdopaminergia and frontal hypo-dopaminergia (Davis et al., 
1991). In light of these data, a simple interpretation of our finding of additive effects of COMT 158 and alcoholism history on BOLD and frontal task performance is that sober alcoholics express relatively more cortical DA depletion, which is COMT independent. DA pharmacoimaging data pertinent to this issue remains conflicting and difficult to interpret (Cropley et al., 2006); however, a marker for central DA activity [plasma homovanillic acid (Amin et al., 1992)] is depressed in sober alchoholics independent of COMT genotype (Kohnke et al., 2003), formally allowing for this possibility.

In summary, we have identified brain regions in which activity during decision making scales with individual tendency to select smaller, immediate, or larger delayed monetary rewards. Because activity in these areas differed in AA and CS subjects, these differences may contribute to decision-making impairment among alcoholics, representing an intermediate phenotype. Moreover, the finding that the COMT genotype at the Val158Met locus predicted both decision-making behavior and underlying frontoparietal activity points to a therapeutic target for $158^{\mathrm{Val} / \mathrm{Val}}$ individuals struggling to overcome substance abuse disorders. Because regional activation during decision making predictive of immediate reward bias did not divide neatly along limbic/nonlimbic structures, our results do not support the hypothesis that immediate reward bias results from an overactive "hot" limbic system relative to a cool reason circuit. Instead, our results favor the idea that immediate reward bias is attributable to a stronger influence on choice of neural representations of proximate outcome comparisons and that, in contrast, lateral OFC activity represents temporally distal outcome comparisons and contributes to the ability to wait for a larger reward. These results thus suggest that cognitive therapies for substance abuse that focus on strengthening neural representations of long-term outcomes may aid in decision making during recovery.

\section{References}

Amin F, Davidson M, Davis KL (1992) Homovanillic acid measurement in clinical research: a review of methodology. Schizophr Bull 18:123-148.

Balleine BW, Killcross S (2006) Parallel incentive processing: an integrated view of amygdala function. Trends Neurosci 29:272-279.

Barraclough DJ, Conroy ML, Lee D (2004) Prefrontal cortex and decision making in a mixed-strategy game. Nat Neurosci 7:404-410.

Baxter MG, Murray EA (2002) The amygdala and reward. Nat Rev Neurosci 3:563-573.

Berlin HA, Rolls ET, Kischka U (2004) Impulsivity, time perception, emotion and reinforcement sensitivity in patients with orbitofrontal cortex lesions. Brain 127:1108-1126.

Bilder RM, Volavka J, Lachman HM, Grace AA (2004) The catechol-Omethyltransferase polymorphism: relations to the tonic-phasic dopamine hypothesis and neuropsychiatric phenotypes. Neuropsychopharmacology 29:1943-1961.

Blasi G, Mattay VS, Bertolino A, Elvevag B, Callicott JH, Das S, Kolachana BS, Egan MF, Goldberg TE, Weinberger DR (2005) Effect of catechol-Omethyltransferase val158met genotype on attentional control. J Neurosci 25:5038-5045.

Cardinal RN, Pennicott DR, Sugathapala CL, Robbins TW, Everitt BJ (2001) Impulsive choice induced in rats by lesions of the nucleus accumbens core. Science 292:2499-2501.

Chen J, Lipska BK, Halim N, Ma QD, Matsumoto M, Melhem S, Kolachana BS, Hyde TM, Herman MM, Apud J, Egan MF, Kleinman JE, Weinberger DR (2004) Functional analysis of genetic variation in catechol-Omethyltransferase (COMT): effects on mRNA, protein, and enzyme activity in postmortem human brain. Am J Hum Genet 75:807-821.

Cheung TH, Cardinal RN (2005) Hippocampal lesions facilitate instrumental learning with delayed reinforcement but induce impulsive choice in rats. BMC Neurosci 6:36.

Congdon E, Canli T (2005) The endophenotype of impulsivity: reaching consilience through behavioral, genetic, and neuroimaging approaches. Behav Cogn Neurosci Rev 4:262-281.

Cools R, Robbins TW (2004) Chemistry of the adaptive mind. Philos Transact A Math Phys Eng Sci 362:2871-2888.

Cropley VL, Fujita M, Innis RB, Nathan PJ (2006) Molecular imaging of the dopaminergic system and its association with human cognitive function. Biol Psychiatry 59:898-907.

D’Esposito M (2007) From cognitive to neural models of working memory. Philos Trans R Soc Lond B Biol Sci 362:761-772.

Davis KL, Kahn RS, Ko G, Davidson M (1991) Dopamine in schizophrenia: a review and reconceptualization. Am J Psychiatry 148:1474-1486.

De Martino B, Kumaran D, Seymour B, Dolan RJ (2006) Frames, biases, and rational decision-making in the human brain. Science 313:684-687.

De Panfilis C, Schwarzbauer C (2005) Positive or negative blips? The effect of phase encoding scheme on susceptibility-induced signal losses in EPI. NeuroImage 25:112-121.

de Wit H, Enggasser JL, Richards JB (2002) Acute administration of $\mathrm{d}$-amphetamine decreases impulsivity in healthy volunteers. Neuropsychopharmacology 27:813-825.

Dom G, Sabbe B, Hulstijn W, van den Brink W (2005) Substance use disorders and the orbitofrontal cortex: systematic review of behavioural decision-making and neuroimaging studies. Br J Psychiatry 187:209-220.

Dorris MC, Glimcher PW (2004) Activity in posterior parietal cortex is correlated with the relative subjective desirability of action. Neuron 44:365-378.

Egan MF, Goldberg TE, Kolachana BS, Callicott JH, Mazzanti CM, Straub RE, Goldman D, Weinberger DR (2001) Effect of COMT Val108/158 Met genotype on frontal lobe function and risk for schizophrenia. Proc Natl Acad Sci USA 98:6917-6922.

Elliott R, Newman JL, Longe OA, Deakin JF (2003) Differential response patterns in the striatum and orbitofrontal cortex to financial reward in humans: a parametric functional magnetic resonance imaging study. J Neurosci 23:303-307.

Enoch MA, Waheed JF, Harris CR, Albaugh B, Goldman D (2006) Sex differences in the influence of COMT Val158Met on alcoholism and smoking in plains American Indians. Alcohol Clin Exp Res 30:399-406.

Ersche KD, Fletcher PC, Lewis SJ, Clark L, Stocks-Gee G, London M, Deakin JB, Robbins TW, Sahakian BJ (2005) Abnormal frontal activations related to decision-making in current and former amphetamine and opiate dependent individuals. Psychopharmacology (Berl) 180:612-623.

Evenden JL (1999) Varieties of impulsivity. Psychopharmacology (Berl) 146:348-361.

Fellows LK, Farah MJ (2005) Dissociable elements of human foresight: a role for the ventromedial frontal lobes in framing the future, but not in discounting future rewards. Neuropsychologia 43:1214-1221.

Friston KJ, Ashburner J, Frith CD, Poline JB, Heather JD, Frackowiak RSJ (1995) Spatial registration and normalization of images. Hum Brain Mapp 3:165-189.

Goldman-Rakic PS, Muly EC, 3rd, Williams GV (2000) D(1) receptors in prefrontal cells and circuits. Brain Res Brain Res Rev 31:295-301.

Hariri AR, Brown SM, Williamson DE, Flory JD, de Wit H, Manuck SB (2006) Preference for immediate over delayed rewards is associated with magnitude of ventral striatal activity. J Neurosci 26:13213-13217.

Hsu M, Bhatt M, Adolphs R, Tranel D, Camerer CF (2005) Neural systems responding to degrees of uncertainty in human decision-making. Science 310:1680-1683.

Ishiguro H, Haruo Shibuya T, Toru M, Saito T, Arinami T (1999) Association study between high and low activity polymorphism of catechol-Omethyltransferase gene and alcoholism. Psychiatr Genet 9:135-138.

Izquierdo A, Suda RK, Murray EA (2004) Bilateral orbital prefrontal cortex lesions in rhesus monkeys disrupt choices guided by both reward value and reward contingency. J Neurosci 24:7540-7548.

Kahn I, Yeshurun Y, Rotshtein P, Fried I, Ben-Bashat D, Hendler T (2002) The role of the amygdala in signaling prospective outcome of choice. Neuron 33:983-994.

Kauhanen J, Hallikainen T, Tuomainen TP, Koulu M, Karvonen MK, Salonen JT, Tiihonen J (2000) Association between the functional polymorphism of catechol-O-methyltransferase gene and alcohol consumption among social drinkers. Alcohol Clin Exp Res 24:135-139.

Kim JW, Park CS, Hwang JW, Shin MS, Hong KE, Cho SC, Kim BN (2006) Clinical and genetic characteristics of Korean male alcoholics with and 
without attention deficit hyperactivity disorder. Alcohol Alcohol 41:407-411.

Kish SJ, Shannak K, Hornykiewicz O (1988) Uneven pattern of dopamine loss in the striatum of patients with idiopathic Parkinson's disease. Pathophysiologic and clinical implications. N Engl J Med 318:876-880.

Knutson B, Taylor J, Kaufman M, Peterson R, Glover G (2005) Distributed neural representation of expected value. J Neurosci 25:4806-4812.

Kohnke MD, Wiatr G, Kolb W, Kohnke AM, Schick S, Lutz U, Vonthein R, Gaertner I (2003) Plasma homovanillic acid: a significant association with alcoholism is independent of a functional polymorphism of the human catechol-O-methyltransferase gene. Neuropsychopharmacology 28:1004-1010.

Kreek MJ, Nielsen DA, Butelman ER, LaForge KS (2005) Genetic influences on impulsivity, risk taking, stress responsivity and vulnerability to drug abuse and addiction. Nat Neurosci 8:1450-1457.

Kweon YS, Lee HK, Lee CT, Pae CU (2005) Association study of catecholO-methyltransferase gene polymorphism in Korean male alcoholics. Psychiatr Genet 15:151-154.

Loewenstein G (1996) Out of control: visceral influences on behavior. Org Behav Hum Decision Processes 65:272-292.

London ED, Ernst M, Grant S, Bonson K, Weinstein A (2000) Orbitofrontal cortex and human drug abuse: functional imaging. Cereb Cortex 10:334-342.

Malkoc SA, Zauberman G (2006) Deferring versus expediting consumption: the effect of outcome concreteness on sensitivity to time horizon. J Market Res 43:618-627.

McClure SM, Laibson DI, Loewenstein G, Cohen JD (2004) Separate neural systems value immediate and delayed monetary rewards. Science 306:503-507.

McClure SM, Ericson KM, Laibson DI, Loewenstein G, Cohen JD (2007) Time discounting for primary rewards. J Neurosci 27:5796-5804.

Minzenberg MJ, Xu K, Mitropoulou V, Harvey PD, Finch T, Flory JD, New AS, Goldman D, Siever LJ (2006) Catechol-O-methyltransferase Val158Met genotype variation is associated with prefrontal-dependent task performance in schizotypal personality disorder patients and comparison groups. Psychiatr Genet 16:117-124.

Mitchell JM, Fields HL, D'Esposito M, Boettiger CA (2005) Impulsive responding in alcoholics. Alcohol Clin Exp Res 29:2158-2169.

Mitchell JM, Tavares VC, Fields HL, D’Esposito M, Boettiger CA (2007) Endogenous opioid blockade and impulsive responding in alcoholics and healthy controls. Neuropsychopharmacology 32:439-449.

Mobini S, Body S, Ho MY, Bradshaw CM, Szabadi E, Deakin JF, Anderson IM (2002) Effects of lesions of the orbitofrontal cortex on sensitivity to delayed and probabilistic reinforcement. Psychopharmacology (Berl) 160:290-298.

Montague PR, King-Casas B, Cohen JD (2006) Imaging valuation models in human choice. Annu Rev Neurosci 29:417-448.

Nichols T, Hayasaka S (2003) Controlling the familywise error rate in functional neuroimaging: a comparative review. Stat Methods Med Res 12:419-446.

Nichols TE, Holmes AP (2002) Nonparametric permutation tests for functional neuroimaging: a primer with examples. Hum Brain Mapp 15:1-25.

Nolan KA, Bilder RM, Lachman HM, Volavka J (2004) Catechol O-methyltransferase Val158Met polymorphism in schizophrenia: differential effects of Val and Met alleles on cognitive stability and flexibility. Am J Psychiatry 161:359-361.

Nussbaum S, Liberman N, Trope Y (2006) Predicting the near and distant future. J Exp Psychol 135:152-161.

Padoa-Schioppa C, Assad JA (2006) Neurons in the orbitofrontal cortex encode economic value. Nature 441:223-226.

Pfefferbaum A, Sullivan EV, Mathalon DH, Lim KO (1997) Frontal lobe volume loss observed with magnetic resonance imaging in older chronic alcoholics. Alcohol Clin Exp Res 21:521-529.

Pinel P, Dehaene S, Riviere D, LeBihan D (2001) Modulation of parietal activation by semantic distance in a number comparison task. NeuroImage 14:1013-1026.

Pizzagalli DA, Sherwood RJ, Henriques JB, Davidson RJ (2005) Frontal brain asymmetry and reward responsiveness: a source-localization study. Psychol Sci 16:805-813.

Reynolds B (2006) A review of delay-discounting research with humans: relations to drug use and gambling. Behav Pharmacol 17:651-667.

Roesch MR, Olson CR (2004) Neuronal activity related to reward value and motivation in primate frontal cortex. Science 304:307-310.

Rubinstein A (2003) "Economics and psychology"? The case of hyperbolic discounting. Int Econ Rev 44:1207-1216.

Rudebeck PH, Walton ME, Smyth AN, Bannerman DM, Rushworth MF (2006) Separate neural pathways process different decision costs. Nat Neurosci 9:1161-1168.

Samochowiec J, Kucharska-Mazur J, Grzywacz A, Jablonski M, Rommelspacher H, Samochowiec A, Sznabowicz M, Horodnicki J, Sagan L, PelkaWysiecka J (2006) Family-based and case-control study of DRD2, DAT, 5HTT, COMT genes polymorphisms in alcohol dependence. Neurosci Lett 410:1-5.

Saunders JB, Aasland OG, Babor TF, de la Fuente JR, Grant M (1993) Development of the Alcohol Use Disorders Identification Test (AUDIT): WHO Collaborative Project on Early Detection of Persons with Harmful Alcohol Consumption-II. Addiction 88:791-804.

Schoenbaum G, Roesch M (2005) Orbitofrontal cortex, associative learning, and expectancies. Neuron 47:633-636.

Sery O, Didden W, Mikes V, Pitelova R, Znojil V, Zvolsky P (2006) The association between high-activity COMT allele and alcoholism. Neuroendocrinol Lett 27:231-235.

Shefrin HM, Thaler RH (1988) The behavioral life-cycle hypothesis. Econ Inquiry 26:609-643.

Sugrue LP, Corrado GS, Newsome WT (2004) Matching behavior and the representation of value in the parietal cortex. Science 304:1782-1787.

Tiihonen J, Hallikainen T, Lachman H, Saito T, Volavka J, Kauhanen J, Salonen JT, Ryynanen OP, Koulu M, Karvonen MK, Pohjalainen T, Syvalahti E, Hietala J (1999) Association between the functional variant of the catechol-O-methyltransferase (COMT) gene and type 1 alcoholism. Mol Psychiatry 4:286-289.

Trope Y, Liberman N (2003) Temporal construal. Psychol Rev 110:403-421.

Tunbridge EM, Harrison PJ, Weinberger DR (2006) Catechol-omethyltransferase, cognition, and psychosis: Val158Met and beyond. Biol Psychiatry 60:141-151.

Volkow ND, Fowler JS (2000) Addiction, a disease of compulsion and drive: involvement of the orbitofrontal cortex. Cereb Cortex 10:318-325.

Wang T, Franke P, Neidt H, Cichon S, Knapp M, Lichtermann D, Maier W, Propping P, Nothen MM (2001) Association study of the low-activity allele of catechol-O-methyltransferase and alcoholism using a familybased approach. Mol Psychiatry 6:109-111.

Williams-Gray CH, Hampshire A, Robbins TW, Owen AM, Barker RA (2007) Catechol O-methyltransferase val158met genotype influences frontoparietal activity during planning in patients with Parkinson's disease. J Neurosci 27:4832-4838.

Winstanley CA, Theobald DE, Cardinal RN, Robbins TW (2004) Contrasting roles of basolateral amygdala and orbitofrontal cortex in impulsive choice. J Neurosci 24:4718-4722.

Winterer G, Egan MF, Kolachana BS, Goldberg TE, Coppola R, Weinberger DR (2006a) Prefrontal electrophysiologic "noise" and catechol-Omethyltransferase genotype in schizophrenia. Biol Psychiatry 60:578-584.

Winterer G, Musso F, Vucurevic G, Stoeter P, Konrad A, Seker B, Gallinat J, Dahmen N, Weinberger DR (2006b) COMT genotype predicts BOLD signal and noise characteristics in prefrontal circuits. NeuroImage 32:1722-1732.

Yacubian J, Glascher J, Schroeder K, Sommer T, Braus DF, Buchel C (2006) Dissociable systems for gain- and loss-related value predictions and errors of prediction in the human brain. J Neurosci 26:9530-9537.

Zauberman G, Lynch JG, Jr (2005) Resource slack and propensity to discount delayed investments of time versus money. J Exp Psychol Gen 134:23-37. 